\title{
THE ASYMPTOTICS OF EXTINCTION IN NONLINEAR DIFFUSION REACTION EQUATIONS
}

\author{
R. E. GRUNDY ${ }^{1}$
}

(Received 15 November 1990; revised 12 January 1991)

\begin{abstract}
In this paper we consider the asymptotics of extinction for the nonlinear diffusion reaction equation

$$
\frac{\partial u}{\partial t}=\frac{\partial^{2}\left(u^{m}\right)}{\partial \dot{x}^{2}}-u^{p}, \quad m>1,0<p<1,
$$

with non-negative initial data possessing finite support. For $t>0$, both solution and support vanish as $t \rightarrow T$ and $x \rightarrow x_{0}$. With $T$ as the extinction time we construct the asymptotic solution as $\tau=T-t \rightarrow 0$ near the extinction point $x_{0}$ using matched expansions. Taking $x_{0}=0$, we first form an outer expansion valid when $\eta=x t^{-(m-p) / 2(1-p)}=O(1)$. This is nonuniformly valid for large $|\eta|$ and has to be replaced by an intermediate expansion valid for $|x|=O\left(\tau^{-1 / \ell_{0}}\right)$ where $\ell_{0}$ is an even integer greater than unity. If $p+m \geq 2$ this expansion is uniformly valid while for $p+m<2$, there are regions near the edge of the support where diffusion becomes important. The zero order solution in these inner regions is discussed numerically.
\end{abstract}

\section{Introduction}

In this paper we consider solutions of the initial value problem with positive initial data for the equation

$$
\frac{\partial u}{\partial t}=\frac{\partial^{2}\left(u^{m}\right)}{\partial x^{2}}-u^{p}
$$

which become identically zero in finite time, a phenomenon known as extinction of the solution. For initial data with finite support, an essential feature of the solutions to (1.1) is the appearance of interfaces which separate regions

\footnotetext{
${ }^{1}$ Department of Mathematical Sciences, University of St. Andrews, Scotland.

(C) Copyright Australian Mathematical Society 1992, Serial-fee code 0334-2700/92
} 
where $u \equiv 0$ from those where $u$ is positive. In this paper, we confine ourselves to the extinction of solutions which have such support and, recalling the results of Kalashnikov [3], [4] that extinction takes place in finite time if $m>1$ and $0<p<1$, we restrict ourselves to this region of parameter space throughout this paper.

The phenomenon of extinction for initial data with finite support is well illustrated by an exact solution for the case $p+m=2$ due to Kersner [5]. This can be written as

$$
\begin{aligned}
u(x, t)= & (m-1)^{-1 /(m-1)}\left\{\frac{2 m(m+1) t}{(m-1)^{2}}+1\right\}^{-1 /(m-1)} \\
\times & {\left[\frac{\left\{(m-1)^{4}+4 m^{2}\right\}}{4 m^{2}}\left\{\frac{2 m(m+1) t}{(m-1)^{2}}+1\right\}^{2 / m+1}\right.} \\
& \left.-\frac{(m-1)^{4}}{4 m^{2}}\left\{\frac{2 m(m+1) t}{(m-1)^{2}}+1\right\}^{2}-x^{2}\right]^{1 / m-1},
\end{aligned}
$$

whenever the quantity in the large brackets is positive, and $u(x, t)=0$ where it is negative. This solution therefore has the initial data

$$
u(x, 0)=u_{0}(x)= \begin{cases}(m-1)^{-1 / m-1}\left(1-x^{2}\right)^{1 / m-1}, & |x|<1 \\ 0, & |x|>1\end{cases}
$$

with $\frac{\partial\left(u^{m}\right)}{\partial x}$ continuous everywhere. The solution is symmetric about $x=0$, and the interfaces where $u=0$ are located at

$$
\begin{aligned}
x^{2}(t)= & \left\{\frac{2 m(m+1) t}{(m-1)^{2}}+1\right\}^{2 / m+1} \frac{(m-1)^{4}}{4 m^{2}} \\
& \times\left\{1+\frac{4 m^{2}}{(m-1)^{4}}-\left\{1+\frac{2 m(m+1) t}{(m-1)^{2}}\right\}^{2 m / m+1}\right\},
\end{aligned}
$$

which is positive for $0<t<T$, with $|x(t)| \rightarrow 0$ as $t \rightarrow T$, where

$$
T=\frac{(m-1)^{2}}{2 m(m+1)}\left\{\left[1+\frac{4 m^{2}}{(m-1)^{4}}\right]^{(m+1) / 2 m}-1\right\}
$$

is called the extinction time and $x=0$ the extinction point. Thus as $t \rightarrow T$ the interfaces contract around the single isolated extinction point at the origin. We shall return to this exact solution later in the paper. More generally, if $u(x, t)=0$ for $t \geq T>0$, we call $T$ the extinction time and an extinction point is defined as one for which a neighbourhood always exists where 
$u(x, t)>0$ as $t \rightarrow T$. The aim of this paper is to describe, in general, the structure of the solution and behaviour of the interfaces as $t \rightarrow T$ near an extinction point. We are guided in this task by the exact solution (1.2) of Kersner and by previous work on the $m=1$ case by Herraro and Friedman [2] and by Galaktionov, Herraro and Velazquez [1].

The case $m=1$ is important to us, since the results which have been obtained for this value of $m$ give us an indication of what may happen in the case $m>1$. For $m=1,0<p<1$, Herrero and Friedman [2] showed that for certain initial data the extinction points are isolated, and that near such a point, say $x_{0}$,

$$
u(x, t) \sim(1-p)^{\frac{1}{1-p}}(T-t)^{\frac{1}{1-p}}
$$

as $t \rightarrow T,\left|x-x_{0}\right| \leq C(T-t)^{1 / 2}$.

In order to study extinction, therefore, this result suggests we write (1.1), with $m=1$, in terms of the similarity variable $\eta=\left(x-x_{0}\right) /(T-t)^{1 / 2}$ and a new independent variable $v$ where

$$
u(x, t)=\tau^{\frac{1}{1-p}} v(\eta, \tau), \quad \tau=T-t .
$$

If we make this change of variable, then (1.1), with $m=1$, becomes

$$
\frac{\partial v}{\partial \tau}+\tau^{-1}\left\{\frac{\partial^{2} v}{\partial \eta^{2}}-\frac{\eta}{2} \frac{\partial v}{\partial \eta}+\frac{v}{(1-p)}-v^{p}\right\}=0 .
$$

For small $\tau$, the behaviour (1.5) is given by the constant solution to

$$
\frac{\partial^{2} v}{\partial \eta^{2}}-\frac{\eta}{2} \frac{\partial v}{\partial \eta}+\frac{v}{(1-p)}-v^{p}=0
$$

namely $v=(1-p)^{\frac{1}{1-p}}$.

To get an idea of what can happen for $m>1$, we can go through a similar procedure to obtain the counterpart of (1.5). For if we now put

$$
\eta=\left(x-x_{0}\right) / \tau^{\delta} \text { and } u(x, t)=\tau^{\alpha} v(\eta, \tau),
$$

then choosing $\alpha=1 /(1-p), \delta=(m-p) / 2(1-p)>0$, we get an equation of the form

$$
\frac{\partial v}{\partial \tau}+\tau^{-1}\left\{\frac{\partial^{2}\left(v^{m}\right)}{\partial \eta^{2}}-\frac{m-p}{2(1-p)} \eta \frac{\partial v}{\partial \eta}+\frac{v}{(1-p)}-v^{p}\right\}=0 .
$$

Equating the term in brackets to zero, the constant solution again gives $v=$ $(1-p)^{\frac{1}{1-p}}$. This result guides us towards the fundamental assumption that for $m>1,0<p<1$, 
$v \sim(1-p)^{\frac{1}{1-p}}(T-t)^{\frac{1}{1-p}} \quad$ as $t \rightarrow T, \eta=\left(x-x_{0}\right) /(T-t)^{(m-p) / 2(1-p)}=O(1)$,

which is the ansatz on which we base our asymptotic theory. We note in passing that the exact solution of Kersner has the asymptotic form (1.7). We delay further details on this until Section 2.

The plan of the paper is as follows. In Section 2.1, we construct the outer expansion for $u(x, t)$ as $t \rightarrow T$ and $x=O\left(\tau^{(m-p) / 2(1-p)}\right)$. In Section 2.2, we indicate how this expansion breaks down for large values of the outer variable, and at the same time form the intermediate expansion which replaces it. In Section 2.3, the nonuniformity of the intermediate expansion near the edges of the support is discussed. For $p+m<2$, an inner expansion is constructed near each interface, and a numerical procedure for solving the subsequent ordinary boundary value problem is presented. By way of a conclusion, we summarise the results in Section 3, and in an Appendix we discuss the nonexistence of logarithmic type error terms in the outer expansion. This is important since Galaktionov, Herraro and Velasquez (1990) show that such terms do occur for the linear diffusion $(m=1)$ case; in the Appendix we discount this possibility.

\section{The asymptotic solution}

\subsection{The outer expansion}

We consider the initial value problem for the equation

$$
\frac{\partial u}{\partial t}=\frac{\partial^{2}\left(u^{m}\right)}{\partial x^{2}}-u^{p}, \quad 0<p<1, m>1,
$$

and suppose that the solution becomes extinct at $t=T>0$. Without loss of generality, we take the extinction point to be at $x=0$. Making the change of variable indicated in the introduction, namely

$$
u=\tau^{\frac{1}{1-p}} v(\eta, \tau), \quad \tau=T-\tau
$$

and

$$
\eta=x / \tau^{(m-p) / 2(1-p)}
$$

we then have

$$
\frac{\partial^{2}\left(v^{m}\right)}{\partial \eta^{2}}+\tau \frac{\partial v}{\partial \tau}-\frac{(m-p)}{2(1-p)} \eta \frac{\partial v}{\partial \eta}+\frac{v}{(1-p)}-v^{p}=0
$$

For convenience we now put

$$
v=(1-p)^{\frac{1}{1-p}} w \text { and } \eta=\left\{\frac{2 m}{(m-p)(1-p)^{(p-m) /(1-p)}}\right\}^{\frac{1}{2}} \zeta
$$


to give

$$
\frac{\partial^{2}\left(w^{m}\right)}{\partial \zeta^{2}}-m \tau \frac{\partial w}{\partial \zeta}+\frac{2 m(1-p)}{(m-p)} \tau \frac{\partial w}{\partial \tau}+\frac{2 m}{(m-p)}\left(w-w^{p}\right)=0 .
$$

Thus

$$
w=(1-p)^{-1 / !-p} \tau^{-1 / 1-p} u(x, t),
$$

and the fundamental assumption (1.7) can now be recast as

$$
w \sim 1
$$

as $t \rightarrow T, \zeta=O(1)$. Thus we expand

$$
w=1+\sum_{i} M_{i}(\zeta) \tau^{\mu_{i}}, \quad \operatorname{Re}\left(\mu_{i}\right)>0,
$$

as $\tau \rightarrow 0, \zeta=O(1)$. We call (2.9) the outer expansion. We note that, in contrast to the case $m=1$ (Galaktionov et al. [1]) (2.9) asserts that logarithmic terms are specifically excluded in the leading-order perturbation. In fact, logarithmic terms could only conceivably be present if $2(1-p)$ / $(m-p)$ is an integer, which, for $0<p<1$ and $m>1$, could only be so for the case $m+p=2$. We discuss this possibility in the Appendix, where we show that leading-order logarithmic terms cannot occur.

If we now substitute (2.9) into (2.6) and equate coefficients of $\tau^{\mu_{i}}$, we find that the functions $M_{i}(\zeta)$ satisfy

$$
\frac{d^{2} M_{i}}{d \zeta^{2}}-\zeta \frac{d M_{i}}{d \zeta}+\frac{2(1-p)}{(m-p)}\left(1+\mu_{i}\right) M_{i}=0 .
$$

We are now required to find solutions of (2.10) in which exponential growth as $\zeta \rightarrow \pm \infty$ is suppressed. This results in an eigenvalue problem for $\mu_{i}$, and a straightforward calculation reveals that the $M_{i}$ are either odd or even. For the even functions,

$$
\mu_{i}=\frac{n(m-p)}{(1-p)}-1 \text { and } M_{i}(\zeta)=A_{i} H_{2 n}\left(\frac{\zeta}{\sqrt{2}}\right) \quad n=1,2,3, \ldots,
$$

while for the odd functions,

$$
\mu_{i}=\frac{(2 n-1)(m-p)}{2(1-p)}-1
$$

and

$$
M_{i}(\zeta)=B_{i} H_{2 n-1}(\zeta / \sqrt{2}) \quad n=2,3,4, \ldots,
$$

where in the latter case $n=1$ can be included for $m+p>2$. Here the $H_{N}(x)$ are the Hermite polynomials of degree $N$ and argument $x$. We call the $M_{i}(\zeta)$ the eigenfunctions of $(2.10)$ and the $\mu_{i}$ the eigenvalues. We presume that the arbitrary constants $A_{i}$ and $B_{i}$ depend in some way on the 
initial conditions, and of course any number may be zero. Let us suppose that the first nonzero $M_{i}(\zeta)$ be denoted by $\alpha_{0} L_{0}(\zeta)$, and the associated value of $\mu_{i}$ is $\lambda_{0}$; subsequent functions by the sequence $\left\{\alpha_{j} L_{j}(\zeta)\right\}$ and subsequent values of $\mu_{i}$ by $\left\{\lambda_{j}\right\}$. Clearly the $\lambda_{j}$ will be given by

$$
\lambda_{j}=\frac{\ell_{j}(m-p)}{2(1-p)}-1
$$

with

$$
L_{j}=H_{\ell_{j}}(\zeta / \sqrt{2})
$$

where $\ell_{j}$ is a positive integer. Thus the expansion (2.9) can be written as

$$
w=1+\alpha_{0} \tau^{\lambda_{0}} L_{0}(\zeta)+\sum \alpha_{j} \tau^{\lambda_{j}} L_{j}(\zeta)
$$

Of course, interactive terms will appear in (2.13), due to the nonlinearity of the original equation, in addition to the terms we have already identified. These will be of the form

$$
\tau^{k_{1} \lambda_{j}+k_{2} \lambda_{k}} N(\zeta)
$$

where $k_{1}$, and $k_{2}$ are positive integers and $N(\zeta)$ is a polynomial obtained by solving an inhomogeneous ordinary differential equation. It may happen that some of these terms will 'force' the eigenfunctions when $k_{1} \lambda_{j}+k_{2} \lambda_{k}=\lambda_{q}$ for $i, j, k$ and integers $k_{1}$ and $k_{2}$. The leading term of (2.13) will always however be an eigenfunction.

Finally in this section it is instructive to construct here the outer expansion for Kersner's exact solution. For simplicity we take $m=3 / 2$ and $p=1 / 2$, and expand (1.2) as $\tau \rightarrow 0$ and $\zeta=2 x / \tau \sqrt{ } 3=O(1)$ to get

$$
u(\zeta, \tau) \sim \frac{\tau^{2}}{4}\left\{1+\frac{6 \tau}{(1+30 T)}\left(1-\zeta^{2}\right)+\cdots\right\}
$$

which confirms the outer expansion (2.13) and the limiting form (1.7) in this case with $l_{0}=2, \lambda_{0}=1, L_{0}=H_{2}(\zeta / \sqrt{ } 2)$ and $\alpha_{0}=-3 /(1+30 T)$.

\subsection{The intermediate expansion}

We first examine the uniformity of the outer expansion (2.13) as $|\zeta| \rightarrow \infty$. To be specific, let us take $\zeta \rightarrow+\infty$, noting that a similar analysis can be undertaken for $\zeta<0$. Now we know from the definition of the Hermite polynomials that

$$
L_{j} \sim 2^{\ell_{j}}(\zeta / \sqrt{2})^{\ell_{j}}+O\left(\zeta^{\ell_{j-2}}\right), \quad \zeta \rightarrow \infty .
$$

Thus the outer expansion (2.12) is not uniformly valid when $\zeta=O\left(\tau^{-\lambda_{0} / \ell_{0}}\right)$, which suggests we introduce an intermediate variable

$$
\xi=\zeta \tau^{\lambda_{0} / \ell_{0}}
$$


on $(-\infty, \infty)$. Making the change of variable in (2.6) gives

$$
\frac{\tau}{m}\left(\frac{2 \lambda_{0}}{\ell_{0}}\right) \frac{\partial^{2}\left(w^{m}\right)}{\partial \xi^{2}}+\frac{2(1-p)}{(m-p)} \tau \frac{\partial w}{\partial t}-\frac{2(1-p)}{(m-p) \ell_{0}} \xi \frac{\partial w}{\partial \xi}+\frac{2\left(w-w^{p}\right)}{(m-p)}=0,
$$

and writing the outer expansion in terms of $\xi$ in the limit $\zeta \rightarrow+\infty$, yields

$$
w \sim 1+\alpha_{0} 2^{\ell_{0} / 2} \xi^{\ell_{0}}+O\left(\tau^{2 \lambda_{0} / \ell_{0}}\right)+\sum_{i} \alpha_{i} 2^{\ell_{i} / 2} \tau^{\left(\lambda_{i} \ell_{0}-\lambda_{0} \ell_{i}\right) / \ell_{0}}\left\{\xi^{\ell_{i}}+\cdots\right\} .
$$

This indicates that we seek an intermediate expansion

$$
w=W_{0}(\xi)+\tau^{2 \kappa_{0}} W_{1}(\xi)+\tau^{\nu_{1}} W_{2}(\xi)+\cdots
$$

in the limit $\tau \rightarrow 0, \xi=O(1)$, where from (2.16) $\kappa_{0}=\lambda_{0} / \ell_{0}$ and $\nu_{1}=$ $\left(\lambda_{1} l_{0}-\lambda_{0} \ell_{1}\right) / \ell_{0}$. Substituting (2.17) into (2.15) and equating the coefficient of the leading power of $\tau$ to zero gives

$$
\frac{(1-p)}{\ell_{0}} \xi \frac{d W_{0}}{d \xi}=W_{0}-W_{0}^{p},
$$

the solution of which can be written

$$
W_{0}=\left\{1+C \xi^{\ell_{0}}\right\}^{\frac{1}{1-p}}
$$

whenever $1+C \xi^{\ell_{0}}>0$, and zero otherwise, with $C$ an arbitrary constant. Expanding (2.18) for $\xi \rightarrow 0$ and matching with the outer expansion (2.16) gives $C=(1-p) \alpha_{0} 2^{\ell_{0} / 2}$, where for decay with two interfaces we must have $\ell_{0}$ even and $\alpha_{0}<2$. Recalling that

$$
\xi=\zeta \tau^{\kappa_{0}}=x \tau^{\kappa_{0}-\delta}=x(T-t)^{-1 / \ell_{0}},
$$

then to zero order these interfaces are given by

$$
x \sim \xi_{0}(T-t)^{1 / \ell_{0}},
$$

where, from (2.18)

$$
\xi_{0}=\left\{-(1-p) \alpha_{0} 2^{\ell_{0} / 2}\right\}^{-1 / \ell_{0}},
$$

with the appropriate number of roots being taken. Thus where two interfaces are present we may write $(2.18)$, with $\ell_{0}$ even, as

$$
\begin{aligned}
W_{0} & =\left\{1-\left(\frac{\xi}{\xi_{0}}\right)^{\ell_{0}}\right\}^{\frac{1}{1-p}}, & & |\xi|<\xi_{0} \\
& =0, & & |\xi|<\xi_{0} .
\end{aligned}
$$


Going further in (2.17) we find that $W_{1}$ and $W_{2}$ satisfy

$$
\begin{aligned}
\xi \frac{d W_{1}}{d \xi} & -\frac{\ell_{0}}{(1-p)}\left\{(m-p+1)-\frac{2(1-p)}{\ell_{0}}-p W_{0}^{p-1}\right\} W_{1} \\
& =\frac{(m-p) \ell_{0}}{2 m(1-p)} \frac{d^{2}\left(W_{0}^{m}\right)}{d \xi^{2}}
\end{aligned}
$$

and

$$
\xi \frac{d W_{2}}{d \xi}-W_{2}\left\{(1-p) \nu+1-p W_{0}^{p-1}\right\} \frac{\ell_{0}}{(1-p)}=0
$$

\subsection{Uniformity of the intermediate expansion near the interfaces}

It is important now to look at the structure of the intermediate expansion as $\xi \rightarrow \xi_{0}$, since this will reveal any nonuniformities that are present near the interfaces. From (2.23) and (2.24) we can show that in this limit

$$
\begin{aligned}
W_{1} \sim & \frac{-\ell_{0}^{2}(m+p-1)(m-p)}{2 \xi_{0}^{2}(1-p)^{2}(m-1)}\left\{1-\left(\frac{\xi}{\xi_{0}}\right)^{\ell_{0}}\right\}^{\frac{m+p-1}{1-p}}+\cdots \\
& +D_{1}\left\{1-\left(\frac{\xi}{\xi_{0}}\right)^{\ell_{0}}\right\}^{\frac{1}{1-p}}+\cdots
\end{aligned}
$$

and

$$
W_{2} \sim D_{2}\left\{1-\left(\frac{\xi}{\xi_{0}}\right)^{\ell_{0}}\right\}^{\frac{1}{1-p}}+\cdots
$$

Here $D_{1}$ and $D_{2}$ are arbitrary constants, and the first term in (2.25) arises from the particular integral of (2.23); the second term comes from the complementary function. So using (2.25) and (2.26) we can write the expansion (2.17) as $\xi \rightarrow \xi_{0}$ in the form

$$
\begin{aligned}
& w \sim\left\{1-\left(\frac{\xi}{\xi_{0}}\right)^{\ell_{0}}\right\}^{\frac{1}{1-p}} \\
& +\tau^{2 \kappa_{0}}\left[\frac{\ell_{0}^{2}(m-p)(p+m-1)}{2 \xi_{0}^{2}(1-p)^{2}(1-m)}\left\{1-\left(\frac{\xi}{\xi_{0}}\right)^{\ell_{0}}\right\}^{\frac{m+p-1}{1-p}}\right. \\
& \left.+D_{1}\left\{1-\left(\frac{\xi}{\xi_{0}}\right)^{\ell_{0}}\right\}^{\frac{p}{1-p}}+\cdots\right] \\
& +\tau^{\nu}\left[D_{2}\left\{1-\left(\frac{\xi}{\xi_{0}}\right)^{\ell_{0}}\right\}^{\frac{p}{1-p}}+\cdots\right]+\cdots
\end{aligned}
$$


The terms involving the arbitrary constants can be absorbed into the leading approximation by a change of independent variable from $\xi$ to $s$, say, where

$$
s=\xi-D_{1} \frac{(1-p)}{\ell_{0}} \xi_{0}^{\ell_{0}} \xi^{1-\ell_{0}} \tau^{2 \kappa_{0}}-D_{2} \frac{(1-p)}{\ell_{0}} \xi_{0}^{\ell_{0}} \xi^{1-\ell_{0}} \tau^{\nu_{1}}+\cdots
$$

This procedure gives

$$
\begin{aligned}
W(s, \tau) \sim & \left\{1-\left(\frac{s}{\xi_{0}}\right)^{\ell_{0}}\right\}^{\frac{1}{1-p}} \\
& +\frac{\ell_{0}^{2}(m-p)(m+p-1)}{2 \xi_{0}^{2}(1-p)^{2}(1-m)}\left\{1-\left(\frac{s}{\xi_{0}}\right)^{\ell_{0}}\right\}^{\frac{m+p-1}{1-p}} \tau^{i k_{0}}+\cdots
\end{aligned}
$$

This shows clearly that the intermediate expansion is uniformly valid for $m+p>2$, a result that is true in general for $m+p=2$ as well.

It is instructive to confirm this for Kersner's exact solution (1.2) in the following way. Taking $\ell_{0}=2,(2.20)$ gives the intermediate variable as $\xi=x t^{-1 / 2}$ and so writing $u(x, t)$ from (1.2) in terms of $\xi$ and expanding for small $\tau$ gives

$$
\begin{aligned}
& u(\xi, \tau)=\frac{4 \tau^{2}}{(1+30 T)^{2}}\left\{\left[\frac{(1+30 T)}{4}-\xi^{2}\right]^{2}\right. \\
&+\tau\left[\frac{60}{(1+30 T)}\left\{\frac{(1+30 T)}{4}-\xi^{2}\right\}^{2}\right. \\
&\left.\left.-\frac{27}{2}\left\{\frac{(1+30 T)}{4}-\xi^{2}\right\}\right]+\cdots\right\} .
\end{aligned}
$$

The appropriate form for the variable $s$ is given by $s=\xi+27 \tau / 8 \xi+\cdots$, so in terms of $s$ we have

$$
\begin{aligned}
u(s, \tau)=\frac{4 \tau^{2}}{(1+30 T)^{2}}\left\{\left[\frac{(1+30 T)}{4}-s^{2}\right]^{2}\right. & \\
& \left.+\frac{60 \tau}{(1+30 T)}\left[\frac{(1+30 T)}{4}-s^{2}\right]^{2}+\cdots\right\},
\end{aligned}
$$

which is uniformly valid as $s \rightarrow \xi_{0}=(1+30 T) / 4$. We can also confirm the interface behaviour (2.20) for the exact solution by expanding (1.4) for $x^{2}(t)$ as $t \rightarrow T$, giving

$$
x(t) \sim \frac{(1+30 T)^{1 / 2}}{2} \tau^{1 / 2}\left\{1-\frac{27 \tau}{2(1+30 T)}+\cdots\right\} .
$$


Returning now to the general case, we see from (2.28) that for $m+p<2$, the intermediate expansion breaks down when

$$
1-\left(\frac{s}{\xi_{0}}\right)^{\ell_{0}}=O\left(\tau^{\kappa_{1}}\right), \quad \kappa_{1}=2 \kappa_{0}(1-p) /(2-m-p) .
$$

To examine the region of nonuniformity ncar the interface, we rescale $s$ via

$$
s=\xi_{0}-s_{1} \tau^{\kappa_{1}}
$$

and, guided by (2.28), we expand $w$ as

$$
w=\tau^{\kappa_{0} /(1-p)}\left\{U_{0}\left(s_{1}\right)+o(1)\right\}
$$

in the limit, $\tau \rightarrow 0, s_{1}=O(1)$. We call this the inner expansion and $s_{1}$ the inner variable. Rewriting (2.15) in terms of $s_{1}, \tau$ and substituting (2.30) we have to leading order

$$
\frac{d^{2}\left(U_{0}^{m}\right)}{d s_{1}^{2}}+\frac{2 m(1-p) \xi_{0}}{(m-p) \ell_{0}} \frac{d U_{0}}{d s_{1}}-\frac{2 m}{(m-p)} U_{0}^{p}=0
$$

We are required to solve this subject to $U_{0}=0$ and the zero flux condition

$$
\frac{d\left(U_{0}^{m}\right)}{d s_{1}}=0
$$

at some value $s=z^{*}$, say, together with the matching condition with the intermediate solution, namely

$$
\begin{aligned}
U_{0} \sim & \left(\frac{\ell_{0}}{\xi_{0}}\right)^{\frac{1}{1-p}} s_{1}^{\frac{1}{1-p}} \\
& +\frac{\ell_{0}^{2}(m-p)(m+p-1)}{2 \xi_{0}^{2}(1-p)^{2}(1-m)}\left(\frac{\ell_{0}}{\xi_{0}}\right)^{\frac{m+p-1}{1-p}} s_{1}^{\frac{m+p-1}{1-p}}+\cdots, \quad s_{1} \rightarrow \infty .
\end{aligned}
$$

Not surprisingly, since $\xi_{0}$ depends on the initial conditions via $\alpha_{0}$, the constant $\left(\xi_{0} / \ell_{0}\right)$ can be eliminated from $(2.31)-(2.33)$ via the scalings

$$
\begin{aligned}
U_{0} & =\left(\xi_{0} / \ell_{0}\right)^{2 /(m+p-2)} T_{0} \\
s_{1} & =\left(\xi_{0} / \ell_{0}\right)^{(m-p) /(m+p-2)} z
\end{aligned}
$$

to yield (2.31)-(2.33) with $U_{0}$ replaced by $T_{0}$ and $\xi_{0} / \ell_{0}=1$. The condition

$$
T_{0}=\frac{d\left(T_{0}^{m}\right)}{d z}=0
$$

now is applied at $z=z^{*}$, with $z^{*}$ being found as part of the solution. 
A possible numerical scheme for solving the problem for $T_{0}$ and finding $z^{*}$ is outlined below. First we can show that as $z \rightarrow \infty$,

$$
T_{0}=z^{\frac{1}{1-p}}-\frac{(m-p)(m+p-1)}{2(1-p)^{2}(m-1)} z^{\frac{m+p-1}{1-p}}+O\left(z^{\frac{2 m+2 p-3}{p-1}}\right) .
$$

If we now put $z_{1}=z-z^{*}$ then the problem reduces to

$$
\begin{gathered}
\frac{d^{2}\left(T_{0}^{m}\right)}{d z_{1}^{2}}-\frac{2(1-p)}{(m-p)} \frac{d T_{0}}{d z_{1}}-\frac{2 m}{(m-p)} T_{0}^{p}=0 \\
T_{0}=\frac{d\left(T_{0}^{m}\right)}{d z_{1}}=0 \text { at } z_{1}=0
\end{gathered}
$$

and, from (2.35),

$$
T_{0}=z_{1}^{\frac{1}{1-p}}-\frac{(m-p)(m+p-1)}{2(1-p)^{2}(m-1)} z_{1}^{\frac{m+p-1}{1-p}}+\frac{z^{*} z_{1}^{p / 1-p}}{(1-p)} O\left(z^{\frac{2 m+2 p-3}{p-1}}\right), z_{1} \rightarrow \infty .
$$

The ordering indicated in (2.35) is consistent provided $2 m+p<3$, an area in the $(p, m)$ plane which is shown in Figure 1. We can now integrate (2.36) from $z_{1}=0$ using the series

$$
\begin{aligned}
T_{0}= & \left(\frac{m-p}{m+p}\right)^{\frac{1}{m-p}} z_{1}^{\frac{2}{m-p}} \\
& +\left(\frac{m-p}{m+p}\right)^{(2-p) /(m-p)} \frac{2(1-p)(m+p)}{\{p(m+p)-(m+2-p)\}(m-p)} z_{1}^{\frac{4-m-p}{m-p}}+\cdots
\end{aligned}
$$

to initiate the integration. The value of $z^{*}$ which solves the problem can be estimated numerically from $(2.38)$ as

$$
z^{*}=\lim _{z_{1} \rightarrow \infty}\left\{\left(T_{0}\left(z_{1}\right)-z_{1}^{\frac{1}{1-p}}+\frac{(m-p)(m+p-1)}{2(1-p)^{2}(m-1)} z_{1}^{\frac{m+p-1}{1-p}}\right)(1-p) / z_{1}^{\frac{p}{1-p}}\right\} \text {. }
$$

For permitted parameter values outside the range $2 m+p<3$ we would need to extend the series (2.38), but in principle $z^{*}$ could still be estimated using a similar scheme to (2.39). The numerical convergence of the limit (2.39) for $z^{*}$ is shown in Figure 2 (page 426) while the solution in the form of $T_{0} / z_{1}^{1 /(1-p)}$ is shown in Figure 3 (page 426) for parameter values $p=0.5$, $m=1.1$ and $p=0.1, m=1.1$. These results are entirely consistent with the asymptotic expansion (2.35), and we can certainly conclude with a large amount of certainty that a unique solution to the problem exists for the parameter regime $p+m<2$. 


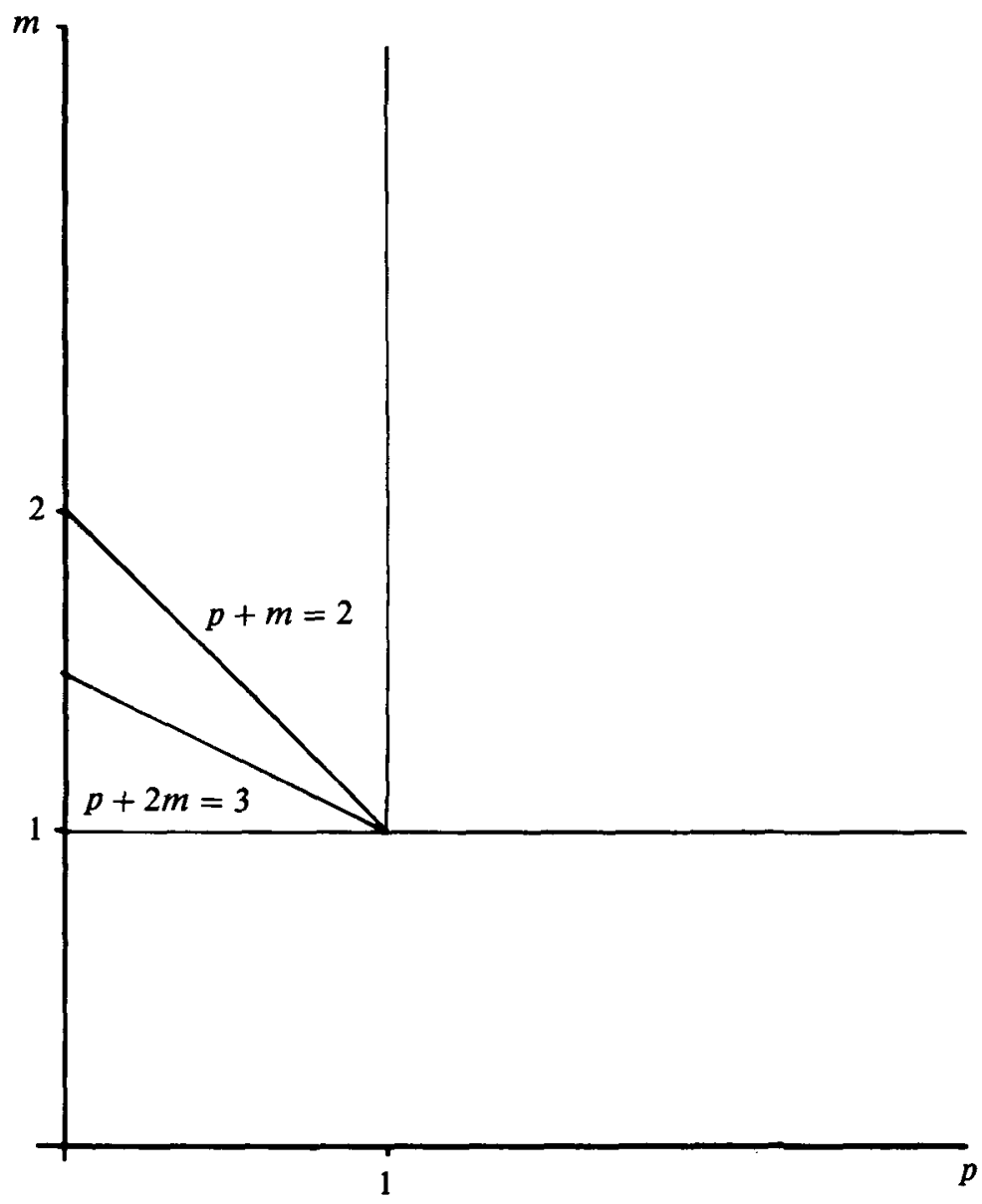

Figure 1. $p-m$ parameter space. 


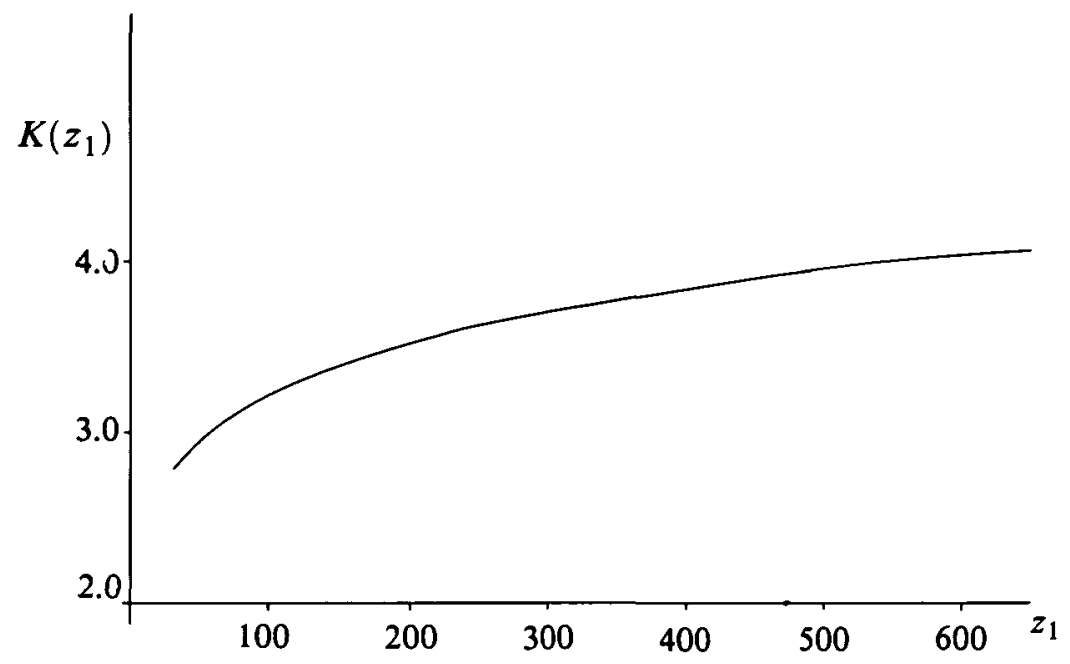

FIGURE 2. $K\left(z_{1}\right)$, defined in the large brackets in (2.39), as a function of $z_{1}$ for $m=1.1$, $p=0.1$. This illustrates the convergence of the limit in (2.39).

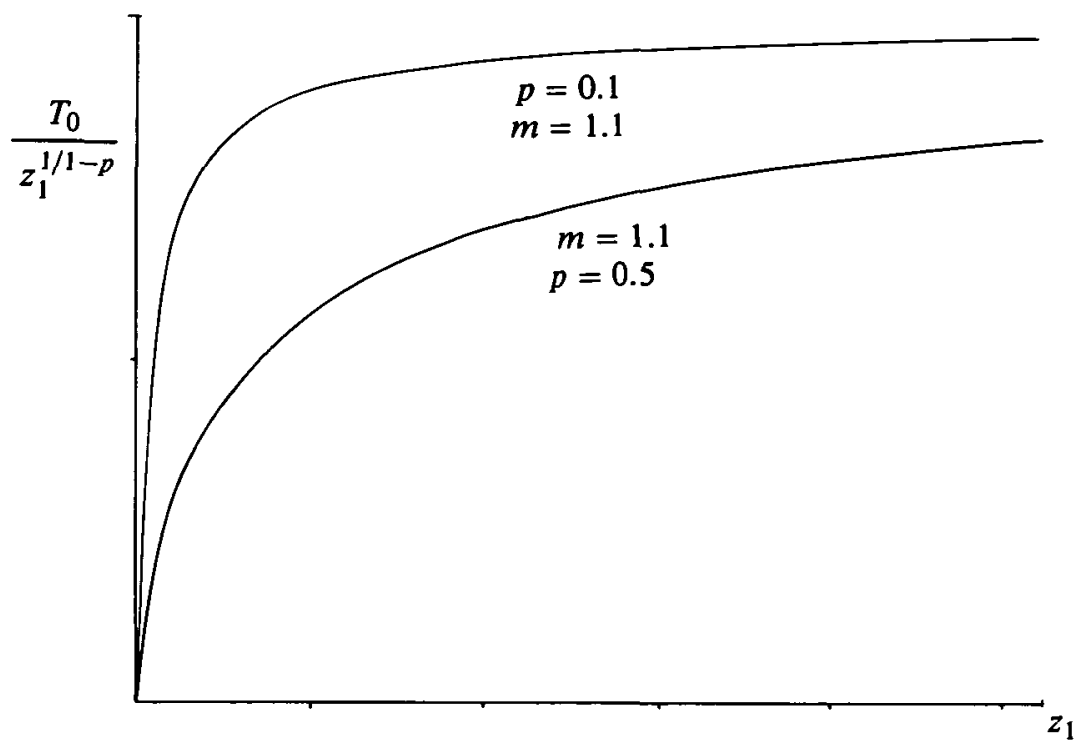
pairs.

FIGURE 3. The solution for $T_{0} z_{1}^{-1 /(1-p)}$ as a function of $z_{1}$ for the indicated parameter 


\section{Summary}

In this paper, we have constructed the analytic behaviour of the solution together with the motion of the interfaces during the final stages of extinction for the nonlinear diffusion-reaction initial-value problem posed by (2.1). With $T$ as the extinction time and the origin as the isolated extinction point, the construction has been developed using matched expansions. An outer expansion valid as $t \rightarrow T$,

$$
\zeta=\left\{\frac{(m-p)(1-p)^{(p-m) /(1-p)}}{2 m}\right\}^{1 / 2} \times(T-1)^{-(m-p) / 2(1-p)}=O(1)
$$

is constructed in the form (2.13), viz.,

$$
u(x, t)=(T-t)^{1 / 1-p}(1-p)^{1 / 1-p}\left\{1+\sum_{j} \alpha_{j} \tau^{\lambda_{j}} L_{j}(\zeta)\right\}
$$

with (2.11) and (2.12). This expansion breaks down as $|\zeta| \rightarrow \infty$ and an intermediate region is defined where $|\xi|=|\zeta| \tau^{\lambda_{0} / \ell_{0}}=O(1)$, together with an intermediate expansion

$$
\begin{aligned}
u(x, t)=(T-t)^{1 / 1-p}(1-p)^{1 / 1-p} & \\
\times & {\left[\left\{1-\left(\frac{s}{\xi_{0}}\right)^{\ell_{0}}\right\}^{1 / 1-p}\right.} \\
& \left.+\frac{(m-p)(m+p-1) \ell_{0}^{2}}{2 \xi_{0}^{2}(1-p)^{2}(1-m)}\left\{1-\left(\frac{s}{\xi_{0}}\right)^{\ell_{0}}\right\}^{m+p-1 / 1-p} \tau^{2 \kappa_{0}}+\cdots\right], \\
\left|s / \xi_{0}\right| \leq 1, &
\end{aligned}
$$

and $u(x, t)=0$ otherwise. Here $s$ is an independent variable defined by (2.27) and for solutions with two interfaces $\ell_{0}$ is an even integer. For $m+p \geq 2$ the expansion is uniformly valid as $s \rightarrow \xi_{0}$. For $m+p<2$ the intermediate expansion itself breaks down as $s \rightarrow \xi_{0}$, and we rescale according to (2.29) as

$$
s_{1}=\left(\xi_{0}-s\right)(T-t)^{-\kappa_{1}}=O(1)
$$

and construct an inner expansion via (2.30) and (2.7) as

$$
u(x, t)=(T-t)^{\left(1+\kappa_{0}\right) /(1-p)}\left\{U_{0}\left(s_{1}\right)+o(1)\right\}
$$

as $t \rightarrow T$. Here

$$
\kappa_{0}=\frac{(m-p)}{2(1-p)}-\frac{1}{\ell_{0}}, \quad \kappa_{1}=\frac{2 \kappa_{0}(1-p)}{(2-m-p)} .
$$


The function $U_{0}\left(s_{1}\right)$ and the value of $s_{1}=z^{*}$ where $U_{0}$ is zero are estimated numerically for selected values of $p$ and $m$. Thus to the appropriate order, $s_{1}=z^{*}$ determines the interface as

$$
s=\xi_{0}+z^{*} \tau^{\kappa_{1}}+o\left(\tau^{\kappa_{1}}\right)
$$

\section{Appendix}

The case $p+m=2$

The aim of the analysis presented in this appendix is to discount the possibility, which happens in the case $m=1$ (Galaktionov et al. [1]), that logarithms may occur in the homogeneous perturbations in (2.8). As we have pointed out, this could only happen if $m+p=2$ and in that event we have to show that an expansion of the form

$$
w=1+\frac{P_{0}(\zeta)}{\log \tau}+\frac{P_{1}(\zeta) \log (\log \tau)}{(\log \tau)^{2}}+\frac{P_{2}(\zeta)}{(\log \tau)^{2}}+\cdots,
$$

with the $P$ 's having algebraic behaviour as $\zeta \rightarrow \pm \infty$, cannot exist. Assuming for the moment that (A.1) is possible then substitution into (2.6) gives

$$
\begin{aligned}
& P_{0}^{\prime \prime}-\zeta P_{0}^{\prime}+P_{0}=0, \\
& P_{1}^{\prime \prime}-\zeta P_{1}^{\prime}+P_{1}=0, \text { and } \\
& P_{2}^{\prime \prime}-\zeta P_{2}^{\prime}+P_{2}=P_{0}+(p / 2) P_{0}^{2}-(1-p)\left(P_{0}^{2}\right)^{\prime \prime}
\end{aligned}
$$

with primes denoting differentiation with respect to $\zeta$. The solutions of (A.2) and (A.3) with algebraic decay as $\zeta \rightarrow \pm \infty$ are simply

$$
P_{0}=A_{0} \cdot \zeta \text { and } P_{1}=A_{1} \cdot \zeta
$$

with $A_{0}$ and $A_{1}$ arbitrary. Thus the equation for $P_{2}(\zeta)$ becomes

$$
P_{2}^{\prime \prime}-\zeta P_{2}^{\prime}+P_{2}=A_{0} \zeta+p A_{0}^{2} \zeta^{2} / 2-2(1-p) A_{0}^{2} .
$$

Differentiating once and solving for $P_{2}^{\prime \prime}$ gives

$$
P_{2}^{\prime \prime}=A_{0} e^{\zeta^{2} / 2} \int_{-\infty}^{\zeta} e^{-\zeta_{1}^{2} / 2} d S_{1}-p A_{0}^{2}+K_{1} e^{\zeta^{2} / 2}
$$

with $K_{1}$ arbitrary. The only way that exponential growth can be suppressed as $\zeta \rightarrow \pm \infty$ is by requiring that $A_{0}=K_{1}=0$. By a similar argument we can show that $A_{1}=0$ and so an expansion of type (A.1) cannot exist. 


\section{References}

[1] V. A. Galaktionov, M. A. Herrero and J. J. L. Velazquez, "The structure of solutions near an extinction point in a semilinear heat equation with strong absorption", Preprint (1990).

[2] M. A. Herraro and A. Friedman, "Extinction properties of semilinear heat equations with strong absorption", J. Math. Anal. and Appl. 124 (1987).

[3] A. S. Kalashnikov, "The propagation of disturbances in problems of nonlinear heat conduction with absorption", USSR Comp. Math. and Math. Physics 14 (1974)

[4] A. S. Kalashnikov, "On the dependence of properties of solutions of parabolic equations in unbounded domains on the behaviour of the coefficients at infinity", Math. USSR Sbornik 53 No. 2 (1986).

[5] R. Kersner, "The behaviour of temperature fronts in media with nonlinear thermal conductivity under absorption", Vestnik Moskovskogo Universiteta. Matematika 33 No. 5 (1978). 